\title{
Principal Component Analysis of the Spectral Energy Distribution and Emission Line Properties of Red 2mass Active Galactic Nuclei
}

\section{Citation}

Kuraszkiewicz, Joanna, Belinda J. Wilkes, Gary Schmidt, Paul S. Smith, Roc Cutri, and Bo? ena Czerny. 2009. "Principal Component Analysis of the Spectral Energy Distribution and Emission Line Properties of Red 2mass Active Galactic Nuclei." The Astrophysical Journal 692 (2) (February 20): 1180-1189. doi:10.1088/0004-637x/692/2/1180.

\section{Published Version}

doi:10.1088/0004-637X/692/2/1180

\section{Permanent link}

http://nrs.harvard.edu/urn-3:HUL.InstRepos:29921899

\section{Terms of Use}

This article was downloaded from Harvard University's DASH repository, and is made available under the terms and conditions applicable to Other Posted Material, as set forth at http:// nrs.harvard.edu/urn-3:HUL.InstRepos:dash.current.terms-of-use\#LAA

\section{Share Your Story}

The Harvard community has made this article openly available.

Please share how this access benefits you. Submit a story.

Accessibility 


\title{
PRINCIPAL COMPONENT ANALYSIS OF THE SPECTRAL ENERGY DISTRIBUTION AND EMISSION LINE PROPERTIES OF RED 2MASS ACTIVE GALACTIC NUCLEI
}

\author{
Joanna Kuraszkiewicz ${ }^{1}$, Belinda J. Wilkes ${ }^{1}$, Gary Schmidt ${ }^{2}$, Paul S. Smith ${ }^{2}$, Roc Cutri ${ }^{3}$, And Bozena Czerny ${ }^{4}$ \\ ${ }^{1}$ Harvard-Smithsonian Center for Astrophysics, Cambridge, MA 02138, USA \\ ${ }^{2}$ Steward Observatory, University of Arizona, Tucson, AZ 85721, USA \\ 3 IPAC, Caltech, MS 100-22, Pasadena, CA 91125, USA \\ ${ }^{4}$ Nicolaus Copernicus Astronomical Center, Warsaw, Poland \\ Received 2008 October 27; accepted 2008 November 5; published 2009 February 23
}

\begin{abstract}
We analyze the spectral energy distributions (SEDs) and emission-line properties of the red $\left(J-K_{S}>2\right.$ ) 2MASS active galactic nuclei (AGNs) using principle component analysis (PCA). The sample includes 44 low redshift AGNs with low or moderate obscuration $\left(\mathrm{N}_{\mathrm{H}}<10^{23} \mathrm{~cm}^{-2}\right)$ as indicated by X-rays and SED modeling. The obscuration of the AGNs allows us to see weaker emission components (host-galaxy emission, AGN scattered light) which are usually outshone by the AGN. The first four eigenvectors explain $70 \%$ of the variance in the sample. Eigenvector 1 (33\% variance in the sample) correlates with the ratios of the intrinsic X-ray flux to the observed optical/IR fluxes and the $F(2-10 \mathrm{keV}) / F([\mathrm{O} \mathrm{III}])$ ratio. We suggest that it is primarily driven by the $L / L_{\text {Edd }}$ ratio and strengthened by intrinsic absorption (both circumnuclear and galactic). Eigenvector 2 (18\% of variance) correlates with optical/IR colors $\left(B-K_{S}, B-R, J-K_{S}\right.$ ) and optical spectral type and depends on the contribution of the host galaxy relative to the observed AGN emission. Eigenvector 3 (12\% of variance) correlates with reddening indicators obtained from the X-rays (hardness ratio, spectral index, $\mathrm{N}_{\mathrm{H}}$ ), and the narrow $\mathrm{H} \alpha / \mathrm{H} \beta$ emission-line ratio. Their relation suggests a common absorber for the optical/X-rays lying outside the narrow-line region possibly in a moderately inclined host galaxy. Eigenvector 4 (8\% of variance) correlates with the degree of polarization and the broad $\mathrm{H} \alpha / \mathrm{H} \beta$ ratio, indicating that dust, which scatters the nuclear emission (continuum and the broad-line region emission), also reddens the broad lines. Our analysis shows that, although as suggested by unification schemes, the inclination dependent obscuration (circumnuclear and the host galaxy) is important in determining the AGN SEDs, the $L / L_{\text {Edd }}$ ratio is the most important factor, followed by host-galaxy emission.
\end{abstract}

Key words: galaxies: active - quasars: general

Online-only material: color figure

\section{INTRODUCTION}

Principal component analysis (PCA) is a mathematical tool used to reduce the dimensionality of a data set without losing information. It transforms the old coordinate system where variables correlate among each other into a new coordinate system, defined by a smaller number of uncorrelated variables called principal components. The first principal component accounts for the most variance in the data, and the successive principal components explain as much of the remaining variance in the data as possible (see e.g., an introduction to PCA by Francis \& Wills 1999 or Murtagh \& Heck 1987).

Since quasi-stellar objects (QSOs) show a plethora of emission-line and continuum properties, PCA is ideal for studying their properties and understanding which are most important. PCA was first introduced to the AGN world by Boroson \& Green (1992; hereafter BG92), who analyzed the optical emission-line and continuum properties of a complete, low redshift, Bright Quasar Survey (BQS) sample. It was found that the primary eigenvector (EV1) is anticorrelated with Fe II $\lambda 4570$ strength (equivalent width and $\mathrm{Fe} \mathrm{II} / \mathrm{H} \beta$ ratio), correlated with [O III] $\lambda 5007$ strength (luminosity and peak) and $\mathrm{H} \beta$ FWHM, and anticorrelated with the blue asymmetry of the $\mathrm{H} \beta$ line. Hence, BG92 concluded that EV1 is driven by a physical parameter that ". . . balances Fe II excitation against the illumination of the narrow-line region." The BG92 EV1 was also found to correlate with the soft $X$-ray properties: luminosity and spectral index (Boroson \& Green 1992; Corbin 1993; Laor et al. 1994, 1997,
Wang et al. 1996; Brandt \& Boller 1998), and the correlations were found to be stronger than with the individual emissionline parameters (Brandt \& Boller 1998). This implied that EV1 has a more fundamental physical meaning. Physical parameters that have been suggested to drive EV1 include: the $L / L_{\mathrm{Edd}}$ ratio, orientation, and black hole spin. Since EV1 is correlated with [O III] emission, thought to be an isotropic property, BG92 concluded that EV1 is not driven by orientation. Although the isotropy of the [O III] emission has since been called into question (Jackson \& Browne 1990; Di Serego Alighieri et al. 1997; Tadhunter et al. 1998) the correlation of EV1 with the inclination independent $\left[\mathrm{O}_{\mathrm{II}}\right] \lambda 3727$ emission, originating at larger distances from the nucleus than [O III], strongly supports the EV1 orientation independence (Kuraszkiewicz et al. 2000), at least for the radio-quiet subsample of the BQS. BG92 suggested that the $L / L_{\text {Edd }}$ ratio was the primary driver of EV1.

BG92 EV1 was also found to correlate with the UV emissionline properties: $\mathrm{C}$ III] width, $\left.\mathrm{Si} \mathrm{III}_{\text {II }} \mathrm{C} \mathrm{III}\right]$ ratio, $\mathrm{CIV}$ and $\mathrm{NV}$ strength, Civ line shift (Wills et al. 1999; Wills et al. 2000; Sulentic et al. 2000), suggesting that EV1 is also linked to the physics of fueling and outflow, and that the higher accretion rates are linked to denser gas and possibly higher nuclear starburst activity (Wang et al. 2006).

By combining observational data together with numerical simulations, Marziani et al. (2001) and Sulentic et al. (2000) were able to reproduce the correlations between optical emission-line properties found in EV1 and confirmed that EV1 was related to $L / L_{\text {Edd }}$, but suggested that, in their sample, 
orientation was also important. Their sample included both the BQS quasars from BG92 (face-on, low obscuration), and inclined/reddened sources of intermediate-type and Type 2 AGN that had FOS/HST spectra.

A different way to approach the analysis of AGN properties is spectral PCA first applied by Francis et al. (1992) to the analysis of more than 200 spectra from the Large Bright QSO Survey (LBQS; redshift range: $0.2 \leqslant z<3.4$ ) or by Shang et al. (2003) to the spectra of PG QSOs. Their analysis was superseded by Yip et al. (2004) who analyzed spectra of more than 16,000 QSOs from the Sloan Digital Sky Survey (SDSS) with a wider range of redshifts $(0<z<5.4)$, inclinations and/or reddening values. Their spectral PCA found the following principal components: mean spectrum, host-galaxy component, UV-optical continuum slope, and correlations of Balmer emission lines. Although the results obtained from spectral PCA and emission line+SED PCA are difficult to compare, it is interesting to invoke them here for comparison with our results.

In this paper, we analyze a sample of red $\left(J-K_{S}>2\right)$ low-redshift 2MASS AGN with moderate absorption $\mathrm{N}_{\mathrm{H}} \lesssim$ $10^{23} \mathrm{~cm}^{-2}$. Their SEDs were analyzed in detail in Kuraszkiewicz et al. (2009; hereafter Paper I), and the emission-line properties are presented in Kuraszkiewicz et al. (2009, in preparation; hereafter Paper II). In this paper, we run PCA on the SED and optical-emission-line-correlation matrix and present our interpretation of the eigenvectors in this moderately-absorbed 2MASS AGN sample. It is important to state here that the results of the PCA are dependent on the selection of the sample and chosen properties. We do not expect the same eigenvectors in the analysis of the 2MASS AGN sample, as those found by BG92 in the unobscured, face-on PG QSO sample. The PG QSOs' first two eigenvectors were dominated by the parameters of the central engine ( $L / L_{\mathrm{Edd}}$ and accretion rate; Boroson 2002), while our 2MASS AGN sample, due to AGN obscuration, is well suited for studies of weaker components such as the host-galaxy emission and intrinsic absorption.

\section{THE SAMPLE AND ITS PROPERTIES}

The sample includes 44 red $\left(J-K_{S}>2\right)$ 2MASS AGNs selected to have $B-K_{S}>4.3$, and $K_{S}<13.8$, and observed by Chandra (see Paper I for more details). The sources have $K$-band luminosities comparable to those of quasars (Cutri et al. 2002) and lie at low redshift $(z<0.37)$. The sample shows a wide range of observed $K_{S}$-band-to-X-ray $(1 \mathrm{keV})$ slopes $(1.1 \lesssim$ $\alpha_{K X} \lesssim 2$; Wilkes et al. 2002), and a broad range of observed polarization fraction at $R$ band $(0 \%<P<13 \%$; Smith et al. 2002, 2003). As found in Paper I, the sample's median SED is redder than that of the blue optically/radio-selected QSOs (Elvis et al. 1994) or the hard-X-ray-selected AGNs (Kuraszkiewicz et al. 2003), showing little or no big blue bump and implying that near-IR color selection isolates the reddest subset of AGNs that can be classified optically. Modeling of the near-IR/optical colors and X-ray spectral analysis in Paper I implies moderate amounts of reddening $\left(\mathrm{N}_{\mathrm{H}}<f e w \times 10^{22} \mathrm{~cm}^{-2}\right)$. The more highly obscured AGNs in this sample are most likely viewed at intermediate angles, where the central engine is obscured from our view by either circumnuclear dust, or dust lying further out in the host galaxy inclined to our line of sight. The obscuration allows us to see/study the contributions of weaker emission components such as host-galaxy emission or AGNscattered light emission, which are normally outshone by the bright AGNs. The sample includes: seven Type 1 AGNs with optical spectra resembling NLS1 or BALQSOs, 11 Type 2 s with
Table 1

Correlation of Eigenvectors with SED and Emission-Line Parameters.

\begin{tabular}{|c|c|c|c|c|}
\hline Variable & $\begin{array}{c}\text { EV1 } \\
32.9 \%\end{array}$ & $\begin{array}{c}\text { EV2 } \\
17.8 \% \\
\end{array}$ & $\begin{array}{c}\text { EV3 } \\
11.6 \% \\
\end{array}$ & $\begin{array}{l}\text { EV4 } \\
8.0 \% \\
\end{array}$ \\
\hline Type & 0.0709 & -0.3933 & 0.0914 & -0.3100 \\
\hline Polarization & -0.0358 & -0.1634 & -0.1641 & 0.4851 \\
\hline$B-R$ & 0.1543 & -0.4196 & -0.0003 & 0.0992 \\
\hline$J-K$ & -0.0563 & -0.3591 & -0.3130 & 0.0368 \\
\hline$B-K$ & 0.0678 & -0.4521 & -0.2056 & 0.0547 \\
\hline$N_{H}$ & -0.0057 & -0.2166 & 0.3696 & -0.0887 \\
\hline$\alpha_{\mathrm{opt}}$ & -0.2108 & 0.3248 & 0.0724 & 0.1596 \\
\hline$\Gamma_{X}$ & 0.0468 & 0.1529 & -0.3814 & -0.2999 \\
\hline $\mathrm{z}$ & -0.0914 & -0.0933 & -0.3250 & 0.4314 \\
\hline $\log F_{1 \mathrm{keV}} / F_{B}$ & 0.4035 & -0.0302 & -0.0100 & 0.0291 \\
\hline $\log F_{1 \mathrm{keV}} / F_{R}$ & 0.3999 & 0.0454 & 0.0172 & 0.0147 \\
\hline $\log F_{1 \mathrm{keV}} / F_{I}$ & 0.3894 & 0.0611 & 0.0415 & 0.0800 \\
\hline $\log F_{1 \mathrm{keV}} / F_{J}$ & 0.3861 & 0.1022 & 0.0230 & 0.0757 \\
\hline $\log F_{1 \mathrm{keV}} / F_{K}$ & 0.3742 & 0.1686 & 0.0862 & 0.0822 \\
\hline Hardness ratio & -0.0520 & -0.2803 & 0.4958 & -0.0517 \\
\hline $\mathrm{H} \alpha^{N L R} / \mathrm{H} \beta^{N L R}$ & -0.1120 & 0.0069 & 0.3347 & 0.1694 \\
\hline $\mathrm{H} \alpha^{B L R} / \mathrm{H} \beta^{B L R}$ & -0.0612 & 0.0165 & 0.2509 & 0.5241 \\
\hline$[\mathrm{O} \mathrm{III}] / F_{2}-10 \mathrm{keV}$ & -0.3531 & -0.0104 & -0.0227 & -0.1378 \\
\hline
\end{tabular}

Note. Parameters that dominate an eigenvector are denoted in bold font.

low (for a Type 2) $\mathrm{N}_{\mathrm{H}} \sim 10^{22} \mathrm{~cm}^{-2}$, and 26 intermediate-type sources. Many of the 2MASS AGNs studied here also show high-optical polarization $(P>4 \%)$.

\section{PRINCIPAL COMPONENT ANALYSIS RESULTS}

In order to investigate the relative importance of various parameters in determining the SED shapes and emission-line properties in the red 2MASS sources, we analyze the relations between the continuum parameters and emission-line parameters by running PCA on the correlation matrix. The first PCA run included all continuum properties analyzed in Paper I (Sections 4, 5, and 6): optical and near-IR colors $\left(B-R, B-K_{S}\right.$, $J-K_{S}$ ), optical type, optical slope, $\alpha_{\text {opt }}$, from optical spectra, degree of polarization, redshift, $X$-ray slope, intrinsic absorption $\mathrm{N}_{\mathrm{H}}$, X-ray hardness ratio, X-ray (1 keV) luminosity (all from Chandra spectral fitting), bolometric luminosity from SEDs, various IR/optical/X-ray flux or luminosity ratios $^{5}$, and the emission-line parameters from Paper II: $W_{\lambda}$, FWHM, shift from systemic redshift of: $\mathrm{H} \beta$ (broad and narrow components), $\mathrm{H} \alpha$ (broad and narrow components), [O III], [O II], optical Fe II, and the following line ratios: $[\mathrm{O} \mathrm{II}] /[\mathrm{O} \mathrm{III}], \mathrm{H} \beta^{\text {broad }} /[\mathrm{O} \mathrm{III}], \mathrm{H} \beta^{\text {narrow }} /[\mathrm{O} \mathrm{III}], \mathrm{H} \alpha^{\text {narrow }} / \mathrm{H} \beta^{\text {narrow }}$, $\mathrm{H} \alpha^{\text {broad }} / \mathrm{H} \beta^{\text {broad }}, \mathrm{Fe}$ II $/ \mathrm{H} \beta^{\text {broad }}, \mathrm{Fe}$ II $/ \mathrm{H} \beta^{\text {narrow }}, \mathrm{Fe}$ II/[O III $]$. A total of 56 parameters were analyzed. The EW, FWHM, line shifts, most line ratios, and optical-to-IR- and IR-to-IR-luminosity ratios did not contribute to the first few eigenvectors, and running PCA with or without them gave similar results (i.e., nearly identical eigenvectors). To simplify the analysis, these parameters were omitted from the final PCA run. The final set of parameters is shown in the first column of Table 1. Columns 2-5 show their relation with the first four eigenvectors (bold font denoting parameters that dominate the eigenvector) and quote in percentages how much variance they explain. Together the first four

\footnotetext{
5 Flux/luminosity ratios included: $F(1 \mathrm{keV}) / F_{B}, F(1 \mathrm{keV}) / F_{R}, F(1 \mathrm{keV}) / F_{I}$, $F(1 \mathrm{keV}) / F_{K}, \mathrm{~L}(0.2-0.4 \mu \mathrm{m}) / \mathrm{L}(0.4-0.8 \mu \mathrm{m}), \mathrm{L}(0.2-0.4 \mu \mathrm{m}) / \mathrm{L}(0.8-$ $1.6 \mu \mathrm{m}), \mathrm{L}(0.4-0.8 \mu \mathrm{m}) / \mathrm{L}(0.8-1.6 \mu \mathrm{m}), \mathrm{L}(1-10 \mu \mathrm{m}) / \mathrm{L}(10-100 \mu \mathrm{m})$, $\mathrm{L}(3-60 \mu \mathrm{m}) / \mathrm{L}(60-100 \mu \mathrm{m}), \mathrm{L}(0.8-1.6 \mu \mathrm{m}) / \mathrm{L}(1-100 \mu \mathrm{m})$, $\mathrm{L}(0.8-1.6 \mu \mathrm{m}) / \mathrm{L}(60-100 \mu \mathrm{m}), \mathrm{L}(0.8-1.6 \mu \mathrm{m}) / \mathrm{L}(3-60 \mu \mathrm{m})$.
} 

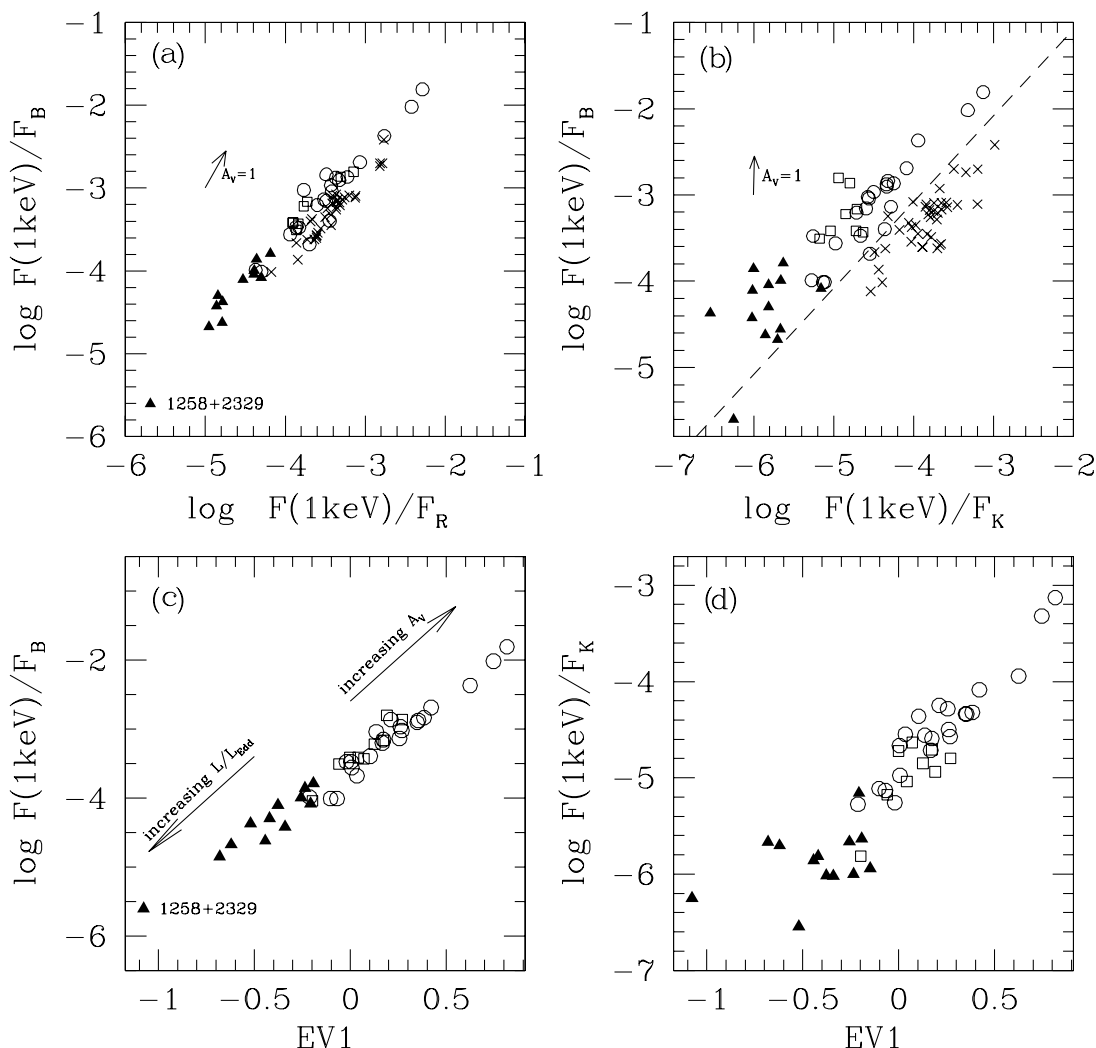

Figure 1. (a, b) Correlations between the intrinsic $1 \mathrm{keV} \mathrm{X}$-ray flux to observed $B$ flux ratio and the intrinsic $1 \mathrm{keV} \mathrm{X-ray} \mathrm{flux} \mathrm{to} \mathrm{observed} R$ and $K$ flux ratios. These are examples of correlations found to compose EV1. The X-ray flux is corrected for Galactic and intrinsic extinction while the $B, R$ and $K$ fluxes are corrected only for Galactic extinction. Crosses represent the blue optically/radio-selected quasars from Elvis et al. (1994). The 2MASS objects in all figures are delineated by their Chandra $\mathrm{S} / \mathrm{N}$ classifications, with circles, squares and filled triangles representing sources with $C$ (highest $\mathrm{S} / \mathrm{N}$ ), $B$ (medium $\mathrm{S} / \mathrm{N}$ ) and $A$ (low $\mathrm{S} / \mathrm{N}$ ) spectral types respectively. The dashed line in Figure 1(b) shows the $B-K_{S}=4.3$ color cut used to select the red 2MASS AGN. 1258+2329 lies below this cut since SuperCOSMOS $B$ magnitude (which is more consistent with the SED) is used here instead of the USNO-A2 magnitude originally used for color selection. (c, d) Correlations between $\mathrm{EV} 1$ and the ratio of intrinsic $1 \mathrm{keV} \mathrm{X}$-ray flux to the observed flux in the $B$ and $K$ bands.

eigenvectors explain $70.3 \%$ of the variance in the sample. Their interpretation is presented below.

\subsection{Eigenvector 1}

Most of the variance $(32.9 \%)$ in the sample is due to the correlations between the ratios of the intrinsic $1 \mathrm{keV} \mathrm{X}$-ray flux to the observed optical/IR flux: $F(1 \mathrm{keV}) / F_{B}, F(1 \mathrm{keV}) / F_{R}$, $F(1 \mathrm{keV}) / F_{I}, F(1 \mathrm{keV}) / F_{J}, F(1 \mathrm{keV}) / F_{K}$ (see Figure 1$)$. We choose to use the observed optical and IR fluxes since, in this moderately obscured/inclined sample, these fluxes depend on intrinsic reddening and host-galaxy emission, which are a priori unknown and hence difficult to subtract. The X-ray fluxes, on the other hand, are corrected for intrinsic absorption, which was estimated from the Chandra X-ray spectral fitting in the following way: the higher $(\gtrsim 80)$ count sources were fit with a power-law and rest-frame absorption with both parameters free (hereafter referred to as the "C" fits), the medium $(\sim 30-80)$ count sources were fit with a fixed power-law with an X-ray photon index $\Gamma=2$ and $N_{H}$ free ("B" fits). For the lowest count sources $(\lesssim 30$ counts; "A" fits), fits were made using a power-law with fixed $\Gamma=2$ and $\mathrm{N}_{\mathrm{H}}=7.6 \times 10^{21} \mathrm{~cm}^{-2}$ (the median absorption from the $C$ fits). The importance of the optical/X-ray flux ratio can be understood, for example, in the disk-wind model paradigm (Murray \& Chiang 1998; Proga et al. 2005 , and references therein), which predict coupling between processes producing X-ray and optical/UV photons. EV1 is also strongly anticorrelated with the $F\left(\left[\mathrm{O}_{\mathrm{III}}\right]\right) / F(2-10 \mathrm{keV})$ ratio (see Figure 2).

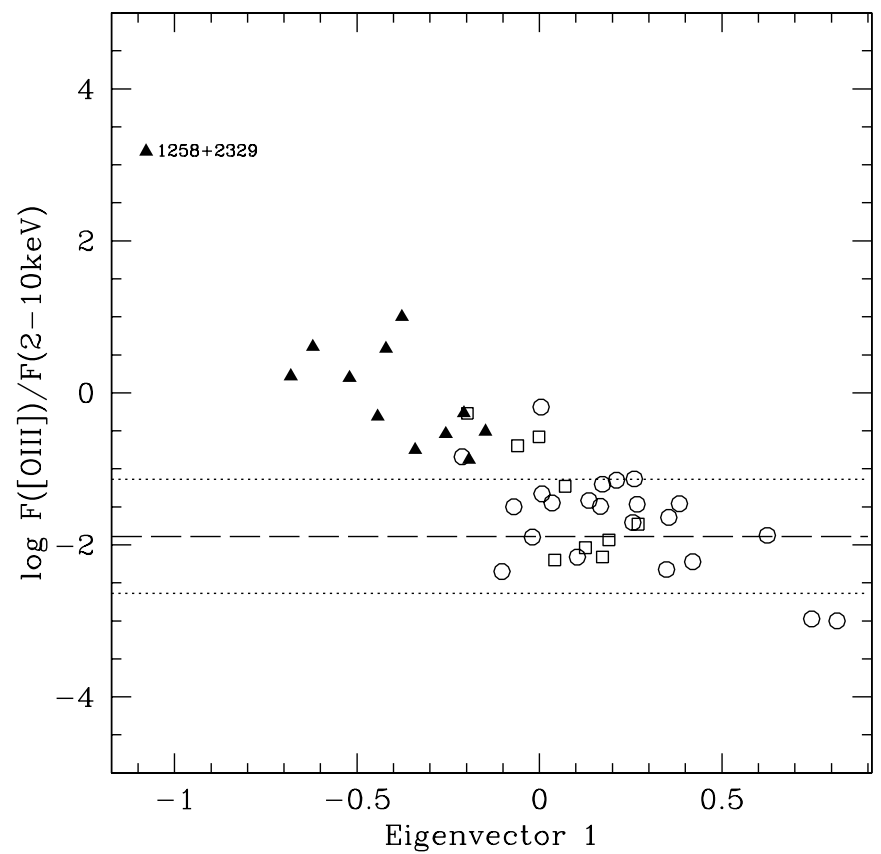

Figure 2. Correlation between the $F([\mathrm{O} \mathrm{III}]) / F(2-10 \mathrm{keV})$ ratio and EV1. X-ray fluxes are corrected for Galactic and intrinsic reddening. The [O III] emissionline flux is corrected for NLR reddening estimated using the narrow $H \alpha / H \beta$ ratio. Symbols indicate $\mathrm{X}$-ray $\mathrm{S} / \mathrm{N}$ as in Figure 1. Dashed line indicates the value of $\log \mathrm{F}\left(\left[\mathrm{O}_{\mathrm{III}}\right]\right) / \mathrm{F}(2-10 \mathrm{keV})=-1.89 \pm 0.75(3 \sigma$ uncertainty-dotted line) found by Mulchaey et al. (1994) for Seyfert 1s and Compton-thin Seyfert 2s. 

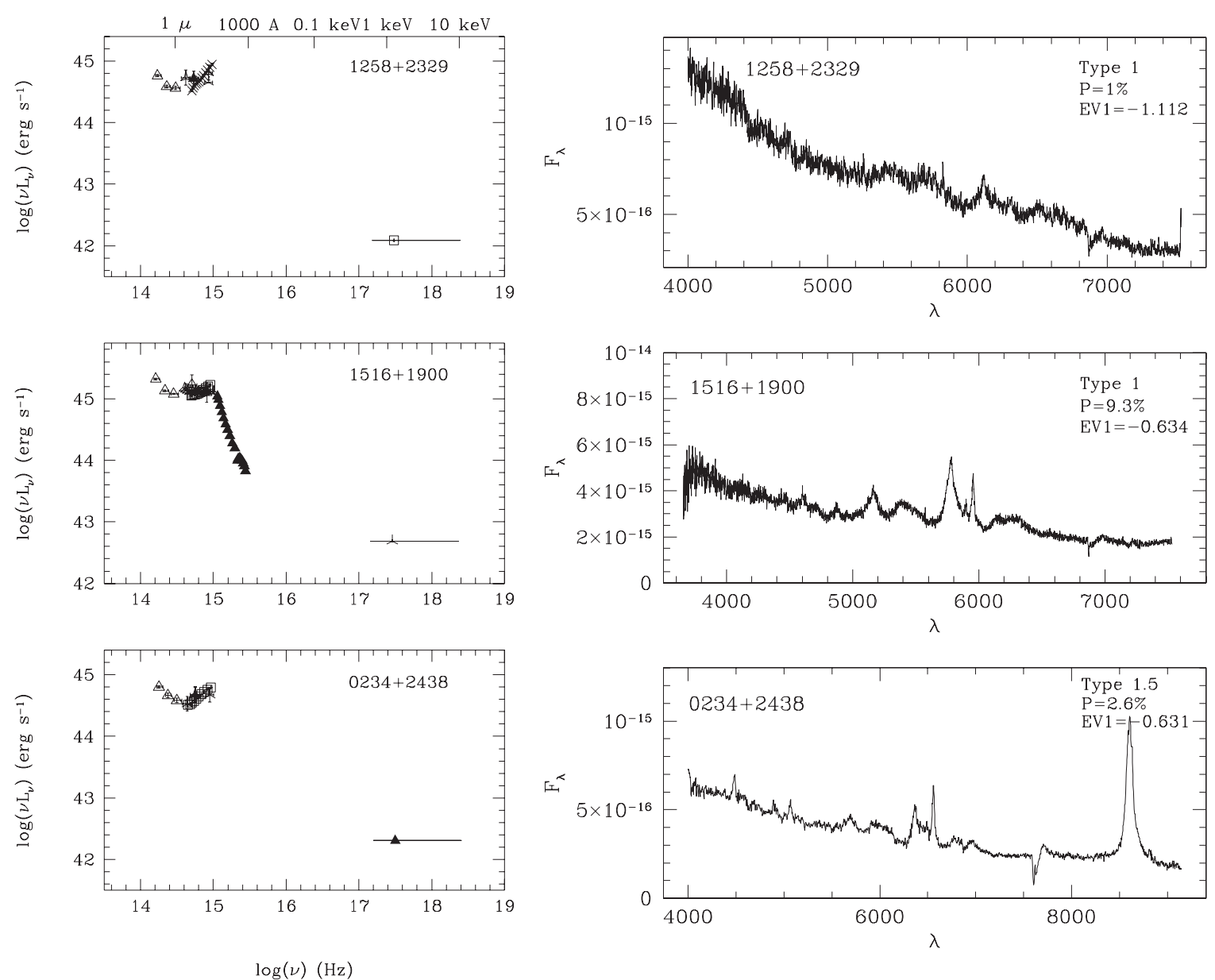

Figure 3. SED and emission-line properties of AGN with the lowest EV1 values. Left column: restframe far-IR to X-ray SEDs. X-ray fluxes are corrected for Galactic and intrinsic absorption. IR-to-UV wavelengths have not been corrected for reddening or host-galaxy contamination. SEDs show low $F(1 \mathrm{keV}) / F_{B, R, J, K}$ ratios and blue $B-R$ colors. Right column: observed-frame optical spectra on $F_{\lambda}\left(\mathrm{erg} \mathrm{s}^{-1} \mathrm{~cm}^{-2} \AA^{-1}\right.$ ) versus $\lambda(\AA)$ scale. Optical spectral type, degree of optical polarization and EV1 value have been denoted on each spectrum. Spectra resemble those of NLS1s and BALQSOs characterized by strong Fe II and weak [O III] emission.

To understand the meaning of EV1 we need to take a closer look at the objects with extreme values of EV1. Their SEDs and optical spectra are presented in Figures 3 and 4, and their properties are summarized in Table 2 . We find that the most negative EV1 sources (Figure 3) with the lowest $F(1 \mathrm{keV}) / F_{B, R, I, J, K}$ and highest $F([\mathrm{O}$ III $]) / F(2-10 \mathrm{keV})$, are Type $1-1.5$, with blue optical spectra dominated by strong Fe II and weak (relatively to $\mathrm{H} \beta$ ) [O III] emission that resemble the spectra of NLS1s and BALQSOs. Although their low S/N $\mathrm{X}$-ray spectra ( $A$ fits) do not yield an estimate for $\mathrm{N}_{\mathrm{H}}$, modeling of $B-R$ and $J-K_{S}$ colors in Paper I (Section 5) gave low $\mathrm{N}_{\mathrm{H}} \lesssim$ $5 \times 10^{21} \mathrm{~cm}^{-2}$ (hence their X-ray fluxes are likely overestimated since $\mathrm{N}_{\mathrm{H}}=7.6 \times 10^{21} \mathrm{~cm}^{-2}$ was assumed for these $A$ fits during spectral fitting) and shows that the SEDs at optical wavelengths are dominated by AGN emission ( $>94 \%$ of the total observed flux at the $R$ band is due to the AGN in these sources).

The most positive EV1 sources (Figure 4) with the highest $F(1 \mathrm{keV}) / F_{B, R, I, J, K}$ and the lowest $F([\mathrm{O} \mathrm{III}]) / F(2-10 \mathrm{keV})$ are Type 1.9-2, with red spectra showing strong [O III] and [O II] emission, and weak Fe II and $\mathrm{H} \beta$ emission and strong galactic features. X-ray spectral fitting gives $\mathrm{N}_{\mathrm{H}} \sim 10^{22} \mathrm{~cm}^{-2}$ consistent with reddening obtained from the modeling of $B-R$ and $J-K_{S}$ colors in Paper I (Section 5) that also shows high host-galaxy contribution at optical wavelengths ( $>61 \%$ of the total observed flux at $R$ band is due to the host galaxy).
The luminosity of the $[\mathrm{O}$ III] $\lambda 5007$ emission line, originating from the narrow-line region (NLR), has been suggested as an indicator of the intrinsic nuclear luminosity of the AGN due to the similarity of the [O III]-to-hard-X-ray flux ratio between Seyfert 1 and Seyfert 2 galaxies (Mulchaey et al. 1994; AlonsoHerrero et al. 1997; Turner et al. 1997). As presented in Figure 2, the most negative EV1 sources do not follow the [O III] to hard$\mathrm{X}$-ray relation showing higher $F([\mathrm{O} \mathrm{III}]) / F(2-10 \mathrm{keV})$ ratios. This indicates that in these sources the intrinsic $\mathrm{X}$-rays are weak. The most positive EV1 sources, on the other hand, have lower $F([\mathrm{O}$ III $]) / F(2-10 \mathrm{keV})$, indicating strong intrinsic X-rays.

The differences in X-ray output relative to optical/UV may be explained for example by an accretion disk and a corona model of Witt et al. (1997). In this model both the accretion disk and the corona accrete and generate energy through viscosity, and division of the flow into optically thin (corona) and optically thick (disk) regions results from the cooling instability discussed by Krolik et al. (1981). The model is defined by three parameters: the mass of the central black hole, the ratio of the luminosity to the Eddington luminosity $L / L_{\mathrm{Edd}}$ (or the accretion rate $\dot{M})$, and the viscosity parameter $\left(\alpha_{\text {vis }}\right)$ assumed to be the same in both the disk and the corona. The model predicts a systematic change in the opt/UV/X-ray SED as a function of $L / L_{\text {Edd }}$. For high $L / L_{\text {Edd }}$, the big blue bump is stronger both in luminosity and relative to the X-rays, so the $F(1 \mathrm{keV}) / F_{B}$ 
Table 2

Objects with Extreme EV1 and EV2 Properties

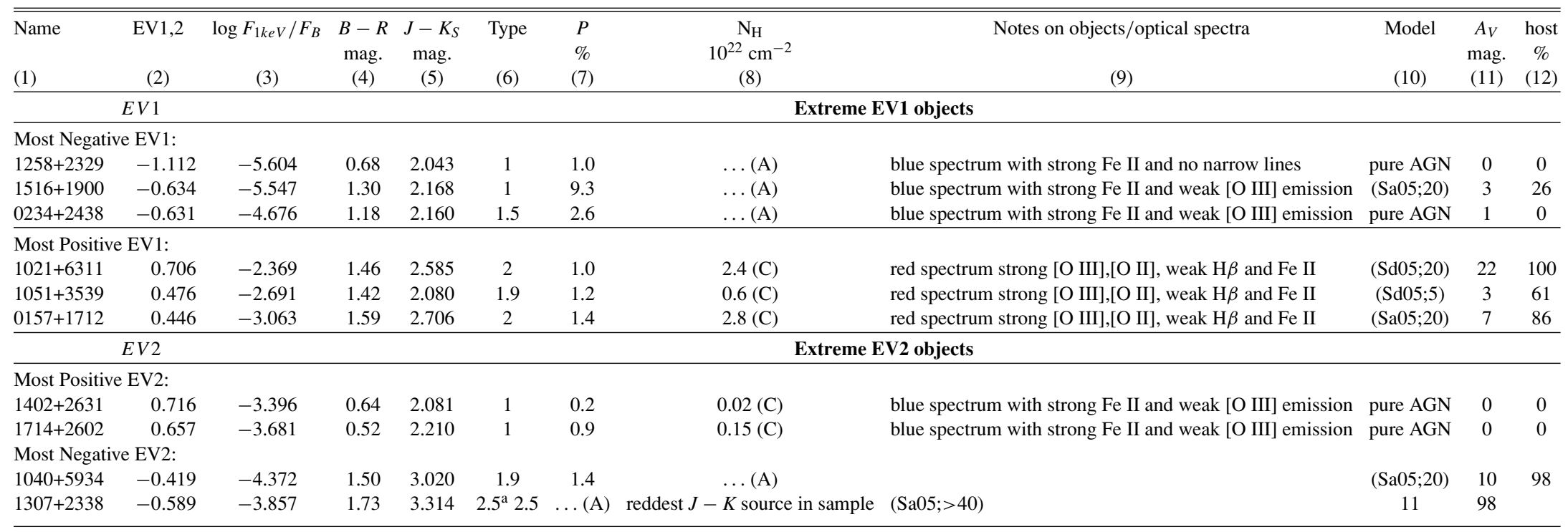

Notes. (1) Object name, (2) EV1 or EV2 value, (3) the ratio of the intrinsic $1 \mathrm{keV}$ flux to the observed flux at the $B$ band, (4), (5) optical and near-IR colors, (6) optical spectral type, (7) degree of linear polarization at the $R$-photometric band, (8) column density measured from Chandra X-ray spectral fitting, in brackets X-ray S/N: A: low, C: high, (9) notes on individual objects and spectra, (10)-(12) parameters obtained from optical/IR color modeling in Paper I: host galaxy type (e.g. (Sd05;5) denotes an Sd host with five Gyr stars and the intrinsic AGN/host-galaxy flux ratio at the $R$ band $=5$ ), AGN reddening and amount of host galaxy relative to the total observed flux at the $R$ band.

${ }^{a}$ Spectrum used to classify the optical type of this source does not extend to $\mathrm{H} \beta$; however $\mathrm{H} \alpha$ emission line is narrow, hence Type 2.5. 

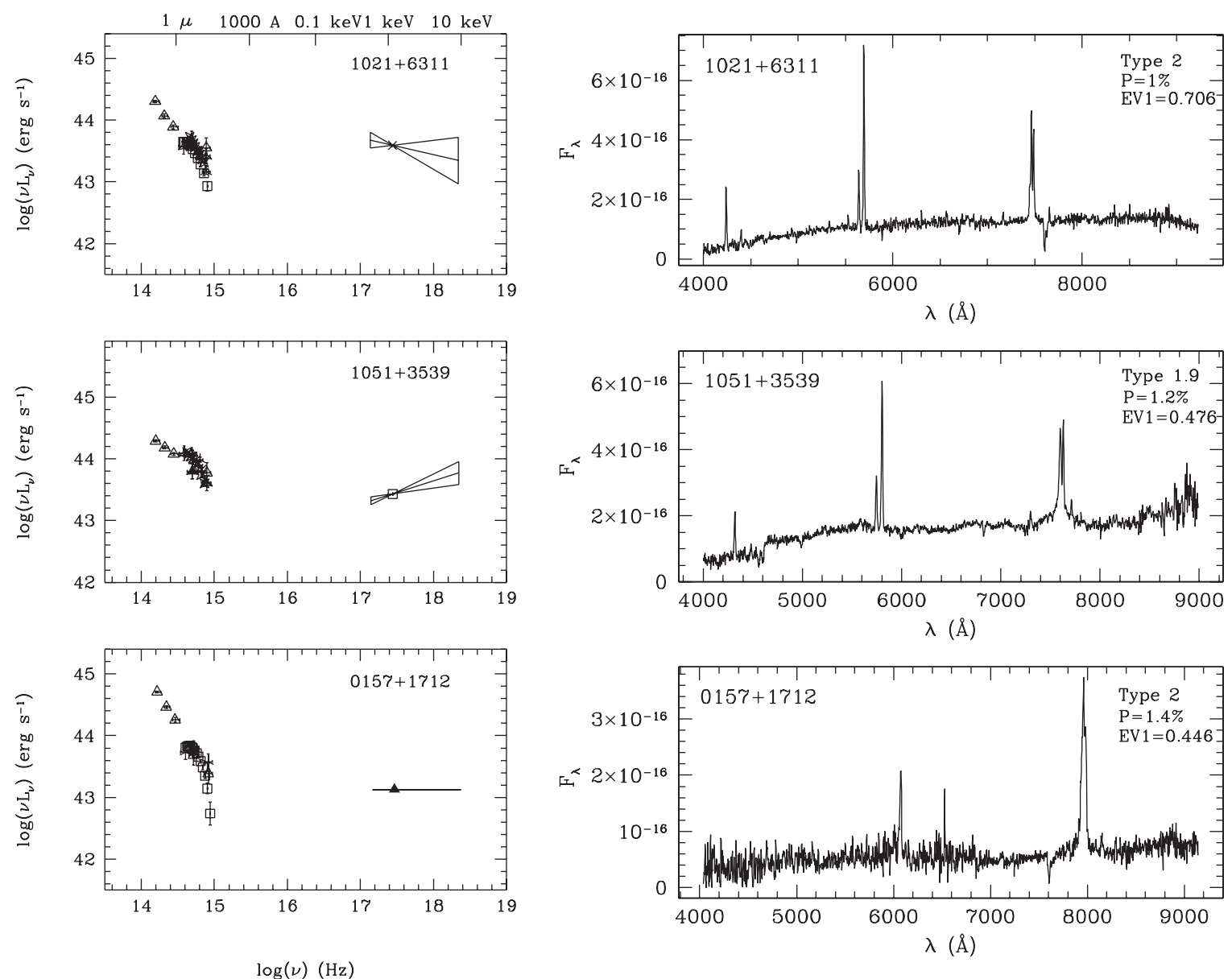

Figure 4. SED and emission-line properties of AGN with the highest EV1 values. Left column: restframe far-IR to X-ray SEDs (for details see Figure 3). SEDs show high $F(1 \mathrm{keV}) / F_{B, R, J, K}$ ratios. Right column: observed-frame optical spectra on $F_{\lambda}\left(\mathrm{erg} \mathrm{s}^{-1} \mathrm{~cm}^{-2} \AA^{-1}\right.$ ) versus $\lambda$ ( $\AA$ ) scale. Optical spectral type, degree of optical polarization and EV1 value have been denoted on each spectrum. Spectra are characterized by strong narrow lines and weak Balmer and Fe II lines.

ratio is low (as in low EV1 sources). For low $L / L_{\text {Edd }}$, the big blue bump is weaker, and the $F(1 \mathrm{keV}) / F_{B}$ ratio becomes high (as in high EV1 sources; see Figure 5). Since the accretion disk and the corona model predictions match those with EV1, they suggest that EV1 is fundamentally related (i.e., anticorrelated) to the $L / L_{\text {Edd }}$ ratio. This is further strengthened by the fact that the most negative EV1 sources (with the lowest $F(1 \mathrm{keV}) / F_{B}$ ) show optical spectra that characterize NLS1s and BALQSOs (strong $\mathrm{Fe}_{\mathrm{II}}$ and weak [O III] emission) that are thought to have high $L / L_{\text {Edd }}$ (e.g., Pounds et al. 1995; Boller et al. 1996; Kuraszkiewicz et al. 2000).

From Table 1 and Figures 1(c), (d), we see that the correlation between EV1 and the $F(1 \mathrm{keV}) / F_{B}$ ratio is stronger than the correlation between EV1 and $F(1 \mathrm{keV}) / F_{K}$. The optical/IR fluxes used to calculate these flux ratios are observed, not intrinsic, so they are affected by dust reddening (and to a lesser extent also by host-galaxy emission). Fluxes at optical wavelengths will be more affected than those at near-IR wavelengths. Since EV1 shows stronger correlation with the $F(1 \mathrm{keV}) / F_{B}$ ratio, which depends both on the $L / L_{\text {Edd }}$ and reddening, and shows more scatter with the $F(1 \mathrm{keV}) / F_{K}$ ratio, which depends mostly on the $L / L_{\text {Edd }}$, and less on reddening, we conclude that EV1 is dominated by $L / L_{\text {Edd }}$. However, dust obscuration also contributes as it stretches the underlying $L / L_{\text {Edd }}$-driven correlation, at optical wavelengths, in these moderately obscured 2MASS sources. If dust obscuration is inclination dependent then this result is consistent with Marziani et al. (2001) who suggest that their EV1 found for a heterogeneous sample of mixed-type (1, 2, and intermediate) AGNs is related to the $L / L_{\text {Edd }}$ ratio convolved with orientation.

\subsection{Eigenvector 2}

Eigenvector 2 (hereafter EV2), which explains $17.8 \%$ of the variance in the sample, is dominated by the observed optical/ IR colors (in order of importance): $B-K_{S}, B-R, J-K_{S}$, and optical spectral type (see Table 1). In Paper I, we found that the optical $B-R$ color, and to a lesser extent the nearIR $J-K_{S}$ color in the 2MASS AGN sample, are affected by reddening and host-galaxy emission (and in a few, highly polarized objects, also by scattered AGN light). We recall the results of the optical/IR color modeling from Paper I in Figure 6. The colors of a pure reddened AGN (median QSO SED from Elvis et al. 1994 reddened by Milky Way dust; long-dash-shortdash line) are modified by host-galaxy emission (Buzzoni 2005 templates), where a range of host-galaxy strengths relative to the intrinsic, unreddened AGN at the $R$ band was assumed (intrinsic AGN/host-galaxy ratio $=5-40$ ). For $A_{V} \lesssim 3$, the optical/near-IR colors are dominated by the AGN. At higher $A_{V}$, the $B-R$ colors become bluer and more consistent with the host-galaxy colors (represented by thick solid lines). The $J-K_{S}$ colors are much less dependent on reddening and host galaxy, only visible if their contribution is strong, i.e., $\mathrm{A}_{V}>10$ and $\mathrm{AGN} /$ host-galaxy ratio low (compare, for example, $(\mathrm{Sd} 05 ; 5)$ and $(\mathrm{Sd} 05 ; 40)$ curves that have a high- and low-host-galaxy contribution respectively). 


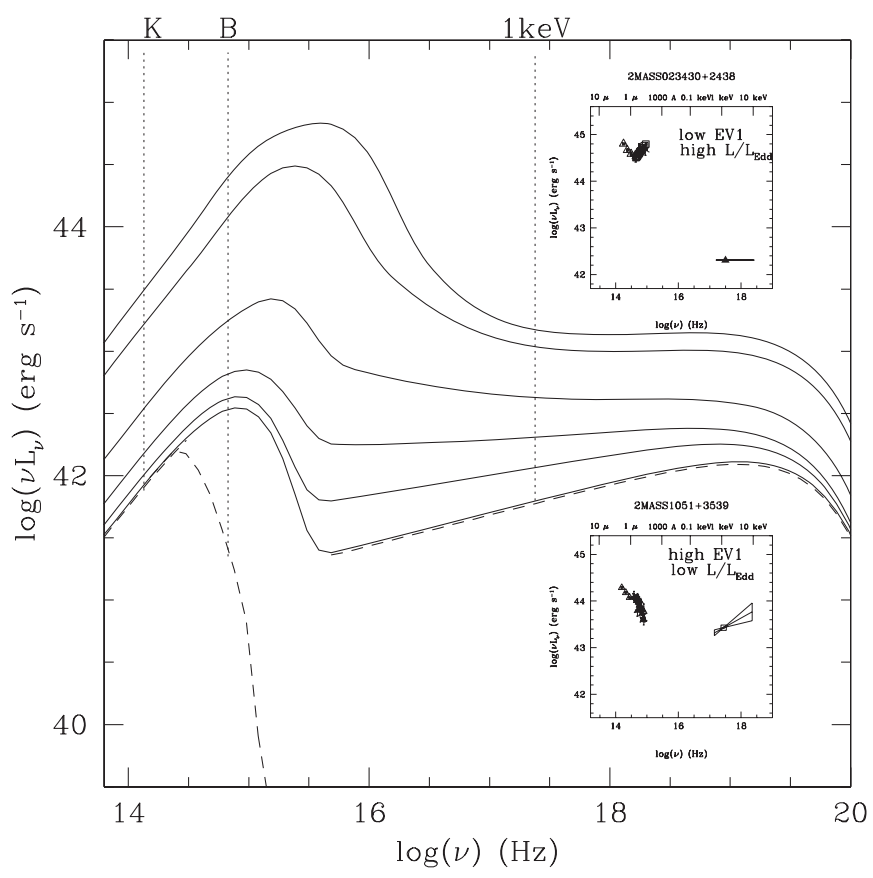

Figure 5. AGN spectral energy distribution predicted by the accretion disk and the corona model of Witt et al. (1997) for a black hole mass $10^{8} M_{\odot}$, viscosity parameter $\alpha=0.33$ and from top to bottom $L / L_{\text {Edd }}=0.25,0.1,0.01$, $0.003,0.0017,0.0013$ respectively. Higher $L / L_{\text {Edd }}$ give a more pronounced big blue bump relative to the X-rays and lower $F(1 \mathrm{keV}) / F_{B, R}$ ratios. Lower $L / L_{\text {Edd }}$ produce a weaker big blue bump relative to the X-rays and higher $F(1 \mathrm{keV}) / F_{B, R}$ ratios. The dashed line shows the $L / L_{\mathrm{Edd}}=0.0013$ model reddened at optical/UV wavelengths by $\mathrm{A}_{V}=3$ (a reddening value obtained from IR/optical color modeling for $1051+3539$, shown in lower insert; X-ray flux is left intrinsic since the Chandra X-ray flux was corrected for both Galactic and intrinsic absorption). Inserts show examples of red 2MASS AGN SEDs with high and low EV1 values.

In Table 2, we summarize the properties of extreme EV2 objects. We find that the most positive EV2 objects are the bluest in $B-R$, implying a strong big blue bump. They also have the bluest $B-K_{S}$ colors in sample. They are Type $1 \mathrm{~s}$, with optical spectra showing strong Fe II and weak [O III] emission, resembling spectra of NLS1 and BALQSO. Column densities derived from X-ray spectral fitting are low $\left(\lesssim 1.5 \times 10^{21} \mathrm{~cm}^{-2}\right)$, and the optical/IR colors are modeled with a pure, unreddened AGN with no host-galaxy contribution.

The most negative EV2 objects are red in $B-K_{S}, B-R$, and $J-K_{S}$ color. The red optical/IR colors are modeled with an AGN reddened by dust with $A_{V}=10-11 \mathrm{mag}$. Such reddening is sufficient to fully obscure the AGN at optical wavelengths, giving way to a large ( $98 \%$ at $R$ band) contribution from the host galaxy. At near-IR wavelengths, the reddened AGN colors still dominate in these sources. The red $J-K_{S}>3$ color can only be produced by a reddened AGN and not the host galaxy which has bluer colors: $J-K_{S}=0.8-1$ (see Figure 6).

In Figure 7, we divide the sources according to the amount of host-galaxy contribution, relative to the total emission at $R$ band, obtained from the optical/IR color modeling (numbers are from Table 7 in Paper I). We find that the amount of hostgalaxy contribution, relative to the reddened AGN decreases with increasing EV2 values. This implies that EV2 depends on the amount of host-galaxy contribution, relative to the observed/ reddened AGN, where at one (negative) end of EV2 reside pure, unreddened AGNs dominating the output at optical/nearIR wavelengths, and on the other (positive) end reside reddened

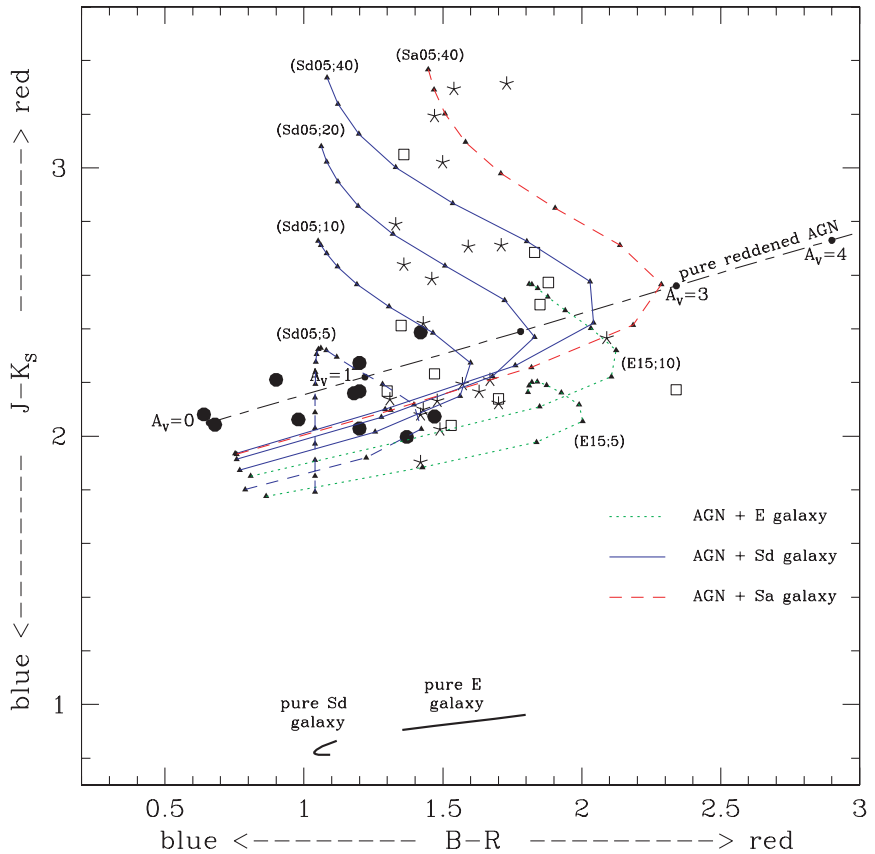

Figure 6. Colors of a reddened AGN combined with contributions from a host galaxy. The long-dash-short-dash curve shows colors of a pure reddened AGN (Elvis et al. 1994 median QSO SED reddened by Milky Way dust). Dotted (green) lines represent AGN colors modified by emission from an elliptical host with a 15 Gyr stellar population (E15; reddest in $B-R$ ). Solid (blue) lines represent an AGN + Sd host and 5 Gyr stellar population (Sd05; bluest in $B-R)$. Short-dashed (red) line: AGN + Sa host galaxy $(B-R$ colors intermediate between E15 and Sd05). Each curve starts at the colors of an unreddened $\left(\mathrm{A}_{V}=0\right) \mathrm{AGN}+$ host galaxy (bluest optical/IR colors) and extends to the reddened $\left(\mathrm{A}_{V}=10\right) \mathrm{AGN}+$ host galaxy in steps of $1 \mathrm{mag}$. denoted by small triangles on the curves (curve $(\mathrm{Sd} 05 ; 5)$ has been extended to $\mathrm{A}_{V}=20$ ). Numbers in parenthesis give the host galaxy type and age of stars followed by the intrinsic, unreddened AGN/host-galaxy flux ratio at $R$ band (e.g., (E15;10): elliptical host, 15 Gyr stars, intrinsic AGN/host $=10$ ). Thick solid curves indicate pure host galaxy ( $\mathrm{E}$ and $\mathrm{Sd}$ ) colors changing with star age (from 1 to $15 \mathrm{Gyr}$ ). Overplotted are the observed colors of the red 2MASS AGNs, divided according to the amount of host-galaxy contribution at the $R$ band obtained from color-color modeling: where $\leqslant 20 \%$ (filled circles), $20 \%-60 \%$ (squares), and $>60 \%$ (stars) of the total observed flux at the $R$ band is due to host-galaxy emission.

(A color version of this figure is available in the online journal.)

AGNs, whose output at optical wavelengths is dominated by host-galaxy emission. A stronger correlation of EV2 with the $B-R$ color than the $J-K_{S}$ color demonstrates that the $B-$ $R$ color is much more sensitive to host-galaxy contamination than the $J-K_{S}$ color, as shown by the color-color modeling (see Figure 6 or detailed discussion in Paper I). Yip et al. (2004), who analyzed the SDSS QSO spectra (sample with a wide range of redshifts, inclination angles, and reddening values) using spectral PCA, also found that their second principle component was related to the host galaxy.

\subsection{Eigenvector 3}

Eigenvector 3 (hereafter EV3; $11.6 \%$ of the variance) is dominated (in order of importance) by the X-ray hardness ratio, $X$-ray spectral index $\Gamma_{X}$, column density $\mathrm{N}_{\mathrm{H}}$ (measured only in the high- and medium-S/N Chandra spectra, i.e., in the $C$ and $B$ fits), and the narrow $\mathrm{H} \alpha / \mathrm{H} \beta$ emission-line ratio. In Figure 8, we show two representative correlations between EV3 and the X-ray hardness ratio and the narrow $\mathrm{H} \alpha / \mathrm{H} \beta$ emission-line ratio. All of these parameters are related to the amount of obscuration 

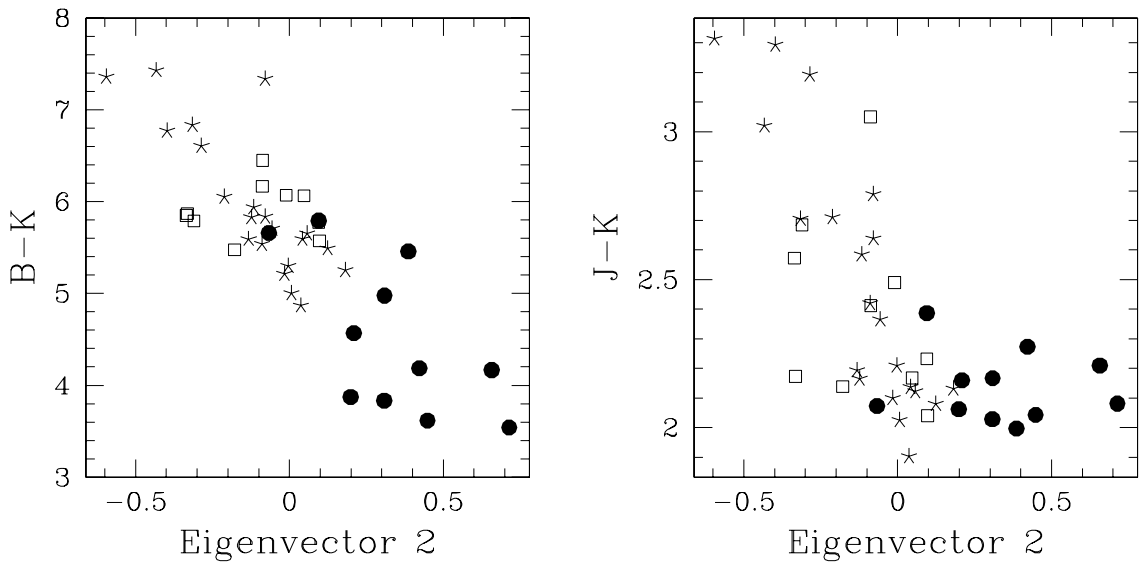

Figure 7. Correlations between EV2 and the $B-K_{S}$ and $J-K_{S}$ colors. Symbols as in Figure 6 denote host-galaxy contribution.
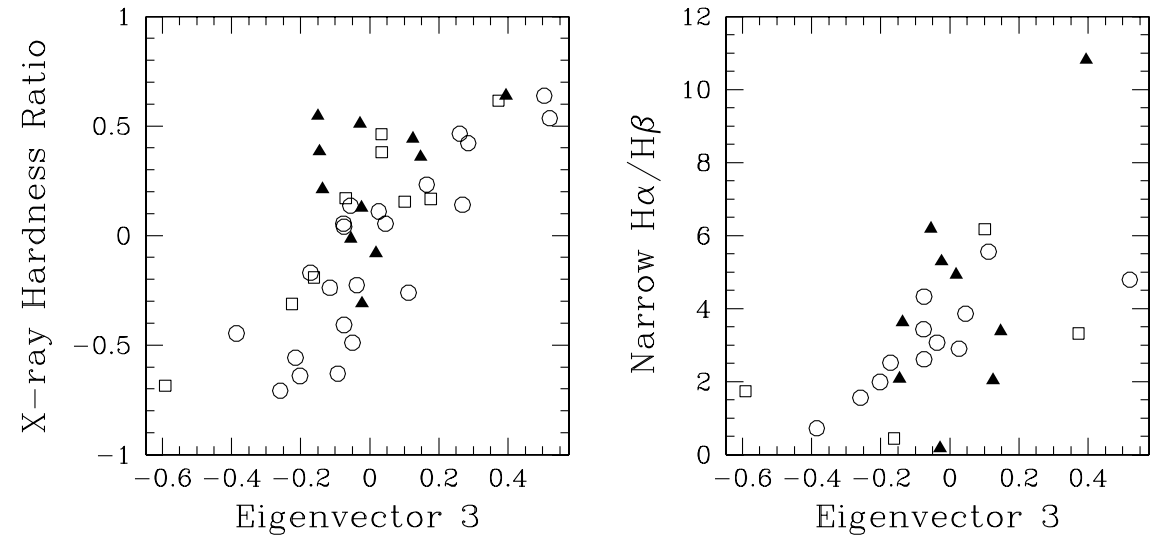

Figure 8. Correlations between EV3 and the X-ray hardness ratio and between EV3 and $\mathrm{H} \alpha / \mathrm{H} \beta$ measured from narrow emission lines. Symbols indicate $\mathrm{X}$-ray $\mathrm{S} / \mathrm{N}$, as in Figure 1.
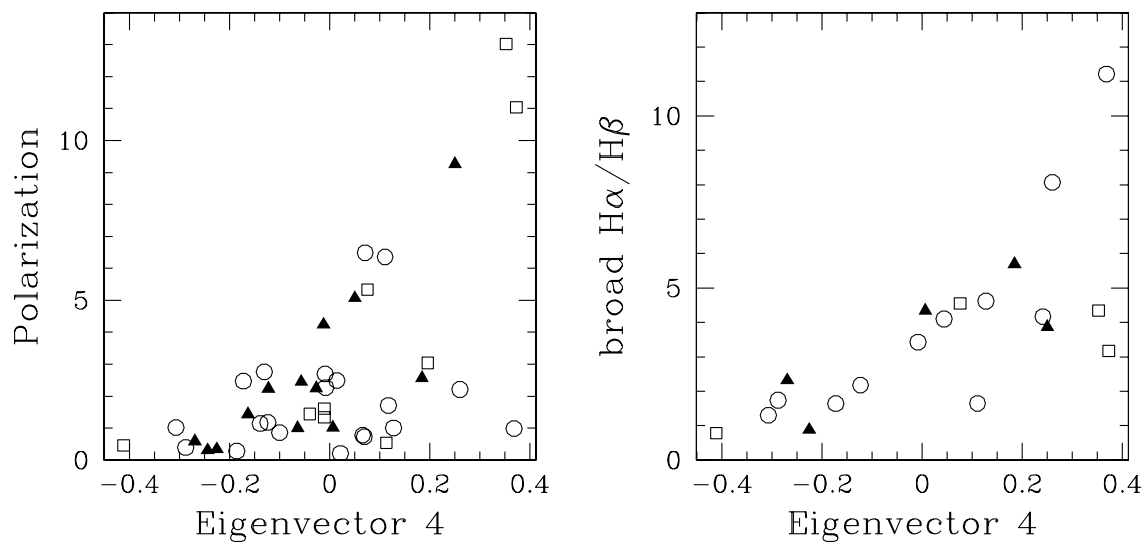

Figure 9. Correlations between EV4 and the degree of optical polarization and broad $\mathrm{H} \alpha / \mathrm{H} \beta$. Symbols indicate X-ray S/N, as in Figure 1.

in X-rays or optical. Their relation implies a common absorber for the optical and X-rays, lying outside the NLR, possibly in a host galaxy inclined to our line of sight (as in Polletta et al. 2008; Deo et al. 2007). This finding is supported by the HST/WFPC2 I-band images of the 2MASS AGNs, which show that these sources reside mostly in host galaxies with inclination angles of $i=50^{\circ}-75^{\circ}$ (mean $i=67^{\circ}$; Marble et al. 2003). We conclude that EV3 is likely dominated by host-galaxy absorption.

The presence of redshift as a component of EV3 (Table 1) is secondary to the presence of $\mathrm{N}_{\mathrm{H}}$, for which measurements become less sensitive, as redshift increases and the absorption is shifted out of the soft X-ray band. The correlation coefficient reduces significantly if $\mathrm{N}_{\mathrm{H}}$ is corrected for redshift (assuming it is intrinsic).

\subsection{Eigenvector 4}

Eigenvector 4 (hereafter EV4; $8.0 \%$ of the variance) is dominated by the degree of optical polarization, broad $\mathrm{H} \alpha / \mathrm{H} \beta$ emission-line ratio, and redshift. The relation between the polarization and the broad $\mathrm{H} \alpha / \mathrm{H} \beta$ ratio implies that the same dust 
scatters the intrinsic continuum+broad lines and reddens the broad emission lines. Since there is no dependence on the narrow $\mathrm{H} \alpha / \mathrm{H} \beta$ ratio in this eigenvector, this dust must lie outside the broad-line region (BLR) but not outside the NLR, i.e., either on the BLR/NLR border, or within the NLR. This is consistent with the conclusions reached by Smith et al. (2003) based on spectropolarimetry of the 2MASS AGNs. The correlation between EV4 and redshift is due to a correlation between redshift and polarization (chance probability $\sim 2 \%$; there is no correlation with the broad $\mathrm{H} \alpha / \mathrm{H} \beta$ ratio-chance probability >60\%). This may be a selection effect as higher redshift sources will have a higher measured degree of polarization due to the wavelength dependence of dust scattering and lower host-galaxy dilution, as one moves into restframe UV where the stellar contribution decreases.

\section{CONCLUSIONS}

PCA of the SED and emission-line parameters of a sample of 44 red $J-K_{S}>2$, moderately obscured $\left(\mathrm{N}_{\mathrm{H}}<10^{23} \mathrm{~cm}^{-2}\right)$ 2MASS AGNs, shows that $70 \%$ of the variance in the sample is explained by four eigenvectors, each having a physical explanation:

1. Eigenvector 1 (33\% of variance in sample) correlates with the intrinsic X-ray to observed optical, IR and [O III] flux ratios, and is strongly related to the $L / L_{\text {Edd }}$ ratio, as shown by disk-corona models, and strengthed by intrinsic reddening.

2. Eigenvector 2 ( $18 \%$ of variance) correlates with optical/IR colors and optical type, and, as shown by the optical/IR color modeling, is related to the relative strength of the host galaxy to the observed/reddened AGNs.

3 . Eigenvector 3 ( $12 \%$ of variance) correlates with reddening indicators, obtained from X-rays and narrow emission lines, indicating a common absorber that affects both the $\mathrm{X}$ rays and narrow emission lines, and probably lies in a moderately inclined host galaxy.

4. Eigenvector 4 ( $8 \%$ of variance) correlates with the degree of polarization and broad $\mathrm{H} \alpha / \mathrm{H} \beta$ ratio, implying the presence of dust (different from the host-galaxy absorber in EV3) that scatters both the BLR and the continuum emission, and reddens the broad lines.

PCA of the SED and/or emission-line properties of unobscured (BG92) and moderately obscured AGNs (Marziani et al. 2001, red 2MASS sample presented here) point to the $L / L_{\text {Edd }}$ ratio as the most important parameter in determining the SED and emission-line properties in AGNs. Since the red 2MASS sample has moderate obscuration, EV1 is also slightly affected by reddening, as is the EV1 found by Marziani et al. (2001) in their heterogeneous sample of moderately obscured AGNs. Successive eigenvectors pick up components depending on the sample chosen: unobscured AGN samples (BG92) probe the properties of the bright central engine (BG92 EV2 is related to accretion rate; see Boroson 2002). Moderately obscured samples, such as the red 2MASS AGNs, pick up weaker components, like the host-galaxy emission, which become important as the reddening obscures the AGN emission. Spectral PCA of the moderately obscured SDSS QSOs (Yip et al. 2004) also finds its EV2 to be related to the host galaxy. Successive eigenvectors in the red 2MASS sample are related to dust obscuration, separated into circumnuclear and host-galaxy absorption. Our analysis is consistent with the orientation-dependent unification scheme for AGNs, where a range of inclination dependent obscuration and host-galaxy contribution (as suggested by Haas et al. 2008) and most importantly $L / L_{\text {Edd }}$ ratios (as suggested by our PCA) can explain the properties of an AGN.

We thank the referee, Gordon Richards, for comments that helped improve the paper and Martin Gaskell for useful discussions. B.J.W. and J.K. gratefully acknowledge the financial support of NASA Chandra grants: GO1-2112A, GO34138A and NASA XMM-Newton grants: NNG04GD27G, NNG05GM24G, which supported various aspects of this work. We also gratefully acknowledge the financial support of grants: NAS8-39073, GO-09161.05-A (HST). P.S.S. acknowledges support from NASA/JPL contract 1256424. This publication makes use of data products from the Two Micron All Sky Survey, which is a joint project of the University of Massachusetts and the Infrared Processing and Analysis Center/California Institute of Technology, funded by the National Aeronautics and Space Administration and the National Science Foundation. We thank F. Murtagh for making his PCA program available on the web.

\section{REFERENCES}

Alonso-Herrero, A., Ward, M. J., \& Kotilainen, J. K. 1997, MNRAS, 288, 977 Boller, Th., Brandt, W. N., \& Fink, H. 1996, A\&A, 305, 53

Boroson, T. A. 2002, ApJ, 565, 78

Boroson, T. A., \& Green, R. F. 1992, ApJS, 80, 109, BG92

Brandt, N., \& Boller, Th. 1998, Astron. Nachr., 319, 163

Buzzoni, A. 2005, MNRAS, 361, 725

Corbin, M. R. 1993, ApJ, 403, L9

Cutri, R., Nelson, B. O., Francis, P. J., \& Smith, P. S. 2002, in Proc. IAU Colloquium 184, ASP Conf. Proc. 284, AGN Surveys, ed. R. F. Green, E. Y. Khachikian, \& D. B. Sanders (San Francisco, CA: ASP), 127

Deo, R. P., Crenshaw, D. M., Kraemer, S. B., Dietrich, M., Elitzur, M., Teplitz, H., \& Turner, T. J. 2007, ApJ, 671, 124

di Serego Alighieri, S., Cimatti, A., Fosbury, R. A. E., \& Hes, R. 1997, A\&A, 328,510

Elvis, M., et al. 1994, ApJS, 95, 1

Francis, P. J., \& Wills, B. J. 1999, in ASP Conf. Ser. 162, Quasars and Cosmology, ed. G. Ferland \& J. Baldwin (San Francisco, CA: ASP), 363

Francis, P. J., Hewett, P. C., Foltz, C. B., \& Chaffee, F. H. 1992, ApJ, 398, 476

Haas, M., Willner, P. S., Heymann, F., Ashby, M. L. N., Fazio, G. G., Wilkes, B. J., Chini, R., \& Siebenmorgen, R. 2008, ApJ, 688, 122

Jackson, N., \& Browne, I. W. A. 1990, Nature, 343, 43

Krolik, J. H., McKee, C. F., \& Tarter, C. B. 1981, ApJ, 249, 422

Kuraszkiewicz, J. K., et al. 2003, ApJ, 590, 128

Kuraszkiewicz, J. K., et al. 2009, ApJ, 692, 1143 (Paper I)

Kuraszkiewicz, J., Wilkes, B. J., Brandt, W. N., \& Vestergaard, M. 2000, ApJ, 542,631

Laor, A., Fiore, F., Elvis, M., Wilkes, B. J., \& McDowell, J. C. 1994, ApJ, 435, 611

Laor, A., Fiore, F., Elvis, M., Wilkes, B. J., \& McDowell, J. C. 1997, ApJ, 477, 93

Marble, A. R., Hines, D. C., Schmidt, G. D., Smith, P. S., Surace, J. A., Armus, L., Cutri, R. M., \& Nelson, B. 2003, ApJ, 590, 707

Marziani, P., Sulentic, J. W., Zwitter, T., Dultzin-Hacyan, D., \& Calvani, M. 2001, ApJ, 558, 553

Mulchaey, J. S., Koratkar, A., Ward, M. J., Wilson, A. S., Whittle, M., Antonucci, R. R. J., Kinney, A. L., \& Hurt, T. 1994, ApJ, 436, 586

Murray, N., \& Chiang, J. 1998, ApJ, 494, 125

Murtagh, F., \& Heck, A. 1987, Multivariate Data Analysis (Berlin, Heidelberg: Springer), ISBN 90-277-2425-3

Polletta, M., Weedman, D., Hönig, S., Lonsdale, C. J., Smith, H. E., \& Houck, J. 2008, ApJ, 675, 960

Pounds, K. A., Done, C., \& Osborne, J. 1995, MNRAS, 277, L5

Proga, D. 2005, ApJ, 630, L9

Shang, Z., Wills, B. J., Robinson, E. L., Wills, D., Laor, A., Xie, B., \& Yuan, J. 2003, ApJ, 586, 52

Smith, P. S., Schmidt, G. D., Hines, D. C., Cutri, R. M., \& Nelson, B. O. 2002, ApJ, 569, 23

Smith, P. S., Schmidt, G. D., Hines, D. C., \& Foltz, C. B. 2003, ApJ, 593, 676 
Sulentic, J. W., Zwitter, T., Marziani, P., \& Dultzin-Hacyan, D. 2000, ApJ, 536, L5

Tadhunter, C. N., Morganti, R., Robinson, A., Dickson, R., Villar-Martin, M. \& Fosbury, R. A. 1998, MNRAS, 298, 1035

Turner, T. J., George, I. M., Nandra, K., \& Mushotzky, R. F. 1997, ApJ, 113, 23

Wang, J., Wei, J. Y., \& He, X. T. 2006, ApJ, 638, 106

Wang, T.-G., Brinkmann, W., \& Bergeron, J. 1996, A\&A, 309, 81
Wilkes, B. J., Schmidt, G. D., Cutri, R. M., Ghosh, H., Hines, D. C., Nelson, B., \& Smith, P. S. 2002, ApJ, 564, L65

Wills, B. J., Laor, A., Brotherton, M. S., Wills, D., Wilkes, B. J., Ferland, G. J., \& Shang, Z. 1999, ApJ, 512, L53

Wills, B. J., Shang, Z., \& Yuan, J. M. 2000, NewAR, 44, 511

Witt, H. J., Czerny, B., \& Zycki, P. T. 1997, MNRAS, 286, 848

Yip, C. W., et al. 2004, AJ, 128, 2603 